INPLASY

PROTOCOL

To cite: Ding et al. The effect of cognitive behavioral therapy

on neuropathic pain: A Metaanalysis. Inplasy protocol

202150102. doi:

10.37766/inplasy2021.5.0102

Received: 29 May 2021

Published: 29 May 2021

Corresponding author:

Anqin Dong

anqindong@163.com

Author Affiliation:

The Fifth Affiliated Hospital of Zhengzhou University

Support: None.

Review Stage at time of this submission: Formal screening of search results against eligibility criteria.

Conflicts of interest:

None declared.

\section{The effect of cognitive behavioral therapy on neuropathic pain: $A$ Meta-analysis}

Ding, N1; Zhang, C2; Zhang, L3; Dong, A4.

Review question / Objective: The aim of this meta-analysis of randomized controlled trials is to evaluate the efficacy and safety of Cognitive behavioral therapy for neuropathic pain. Condition being studied: Neuropathic pain refers to pain caused by injury or dysfunction of the somatosensory system. Epidemiological studies have shown that $20 \%-25 \%$ of the global population suffers from moderate to severe chronic pain, among which the prevalence rate of neuropathic pain is $6.9 \%-10 \%$, which seriously affects their daily activities and quality of life.Cognitive behavior therapy is the general term for a kind of psychotherapy which focuses on correcting and changing patients' adaptations to benign cognition. Its main goal is the remediation of bad cognition, but the patient's Pain symptoms and the emotions can also be changed.

INPLASY registration number: This protocol was registered with the International Platform of Registered Systematic Review and Meta-Analysis Protocols (INPLASY) on 29 May 2021 and was last updated on 29 May 2021 (registration number INPLASY202150102).

\section{INTRODUCTION}

Review question / Objective: The aim of this meta-analysis of randomized controlled trials is to evaluate the efficacy and safety of Cognitive behavioral therapy for neuropathic pain.
Condition being studied: Neuropathic pain refers to pain caused by injury or dysfunction of the somatosensory system. Epidemiological studies have shown that $20 \%-25 \%$ of the global population suffers from moderate to severe chronic pain, among which the prevalence rate of neuropathic pain is $6.9 \%-10 \%$, which 
seriously affects their daily activities and quality of life.Cognitive behavior therapy is the general term for a kind of psychotherapy which focuses on correcting and changing patients' adaptations to benign cognition. Its main goal is the remediation of bad cognition, but the patient's Pain symptoms and the emotions can also be changed.

\section{METHODS}

Search strategy: We will search, with no time restrictions, the following databases for relevant English language literature: PubMed (MEDLINE), the Cochrane Central Register of Controlled Trials (CENTRAL) and Web of Science.The search string will be built as follows: "Cognitive behavioral therapy" AND "Neuropathic pain" AND"Randomized controlled trial". The electronic database search will be supplemented by a manual search of the reference lists of included articles.

Participant or population: Adults with neuropathic pain (as diagnosed by a clinician, or using any recognized diagnostic criteria) will be included.

Intervention: Cognitive behavioral therapy was the main intervention(eg.cognitive therapy, behavior therapy and mindfulnessbased cognitive therapy)

Comparator: Conventional interventions such as nerve block.

Study designs to be included: PubMed, Embase, Cochrane, PsycINFO, Wanfang Database (WANFANG), China Knowledge Network (CNKI), VIP (VIP) and China Biomedical Literature Database (CBM) were electronically searched from the establishment of the database to March 2021 to collect randomized controlled trials (RCT) of the treatment of neuropathic pain. Two researchers independently carried out literature screening, data extraction and methodological quality evaluation, and cross-checked, and used RevMan5.3 software for Meta analysis.
Eligibility criteria: Inclusion criteria: (1) Study type: A Randomized controlled trial (RCT) involving the effect of CBT on pain level and mental health status of patients with neuropathic pain (English or Chinese); (2) Subjects: All patients diagnosed with neuropathic pain; (3) The experimental group received cognitive behavioral therapy, while the control group received other conventional interventions except cognitive behavioral therapy. (4) Outcome indicators: Pain intensity and mental state; (5) Integrity of data: all the included literatures could provide complete baseline information of cases and original data of outcome indicators, or the mean and standard deviation of outcome indicators could be calculated from the data. Exclusion criteria: (1) non-RCT studies or conference reports, non-Chinese or English literature; (2) Animal experiments; (3) Repeated publications in the same study; (4) Overview, experience sharing, case report; (5) unreasonable research of statistical methods and experimental design.

Information sources: We will search articles in four electronic database including PubMed, Embase, Cochrane and PsycINFO. All the English publications until March of 2021 will be searched without any restriction of countries or article type. Reference list of all selected articles will independently screened to identify additional studies left out in the initial search.

Main outcome(s): Pain intensity(PI).

Quality assessment / Risk of bias analysis: The Cochrane Collaborative Network bias risk assessment tool was used to evaluate the methodological quality of the included studies. The bias risk assessment included 6 aspects: selective bias, measurement bias, implementation bias, reporting bias, follow-up bias and other biases.Two researchers independently evaluated the literature quality and cross-checked it. If there were differences in the evaluation results, the third one would be judged by the researchers. 
Strategy of data synthesis: RevMan5.3 software was used for analysis, and the appropriate statistical model was selected according to the heterogeneity test results. Mean difference (MD) and standard mean difference (SMD) were used for pain score and depression scale, and $95 \%$ confidence interval (CI) was used as effect index. Represents the estimated interval of the population parameter, and the test level is $a=0.05$.

Subgroup analysis: There was no subgroup analysis at this time.

Sensitivity analysis: $\mathrm{x} 2$ test was used to test the heterogeneity of the included study results, and 12 was used to determine that when the results were homogenous $(P>0.1$, $12<50 \%$ ), the fixed effect model was used for analysis. If statistical heterogeneity exists $(P \leq 0.1,12 \geq 50 \%)$, then the causes of heterogeneity are analyzed. If clinical heterogeneity exists, subgroup analysis or sensitivity analysis can be performed according to its source, or only descriptive analysis can be performed. If there was no significant clinical heterogeneity, random effects model was used for analysis.

Country(ies) involved: China.

Keywords: Cognitive behavioral therapy; Neuropathic pain; Meta-analysis.

Contributions of each author:

Author 1 - Ning Ding.

Email: 1095262997@qq.com

Author 2 - Cuicui Zhang.

Email: 2971089979@qq.com

Author 3 - Lingxi Zhang.

Email: 445897961@qq.com

Author 4 - Anqin Dong.

Email: anqindong@163.com 